\title{
Design Participativo em Ambientes Distribuídos de Desenvolvimento de Software Educacional Livre: desafios de pesquisa
}

\author{
Daniel Domingos Alves ${ }^{1}$, Ecivaldo de Souza Matos ${ }^{1}$ \\ ${ }^{1}$ Departamento de Ciência da Computação \\ Universidade Federal da Bahia (UFBA) \\ Salvador, Bahia - Brasil \\ \{daniel.domingos, ecivaldo\}@ufba.br
}

\begin{abstract}
Participatory design (PD) in distributed environments is a recent area of research called Distributed Participatory Design. Despite some research efforts in this area, there are still few studies related to this topic. This paper presents the importance and some challenges of PD adoption in the development of free educational software. We also present partial results of a systematic mapping that aims to identify and synthesize studies describing approaches, methods, techniques and tools for PD offree educational software in distributed environments. As a result, we emphasize the relevance of the theme and highlight the challenges and importance of user collaboration in the development of free educational software.
\end{abstract}

Resumo. $O$ design participativo (DP) em ambientes distribuídos é uma área de pesquisa recente denominada Design Participativo Distribuído. Apesar de alguns esforços de pesquisas nessa área, ainda são poucos os estudos relacionados a esse tema. Este artigo apresenta a importância e alguns desafios da adoção do DP no desenvolvimento de software educacional livre. Apresenta-se também resultados parciais de um mapeamento sistemático que visa identificar e sintetizar estudos que descrevem abordagens, métodos, técnicas e ferramentas para o DP de software educacional livre em ambientes distribuídos. Como resultados, enfatiza-se a relevância do tema e destaca-se os desafios e a importância da colaboração de usuários no desenvolvimento de software educacional livre.

\section{Introdução}

Software educacional livre é um programa de computador desenvolvido para fins educacionais, distribuído com o seu código fonte e que permite as "liberdades" de uso, cópia, modificação e redistribuição [Jucá 2006, Stallman 1998]. Os softwares educacionais devem atender aos objetivos educacionais previamente estabelecidos. A participação de especialistas da área de Educação no desenvolvimento de software educacional é necessária e visa garantir a sua efetividade e atender as necessidades pedagógicas [Lucena 1994].

Diversos pesquisadores de Interação Humano-Computador (IHC) têm proposto/aplicado abordagens, métodos e técnicas para envolver os usuários em atividades do processo de design de interação. No entanto, a maioria das abordagens de Design Participativo (DP) é concebida para ter reuniões face a face [Gumm et al. 2006]. 
Além disso, a maioria dos métodos e técnicas de DP é aplicada de forma colocalizada [Gumm et al. 2006, Lazarin and Almeida 2016]. Projetos de software em que os participantes não estão colocalizados podem encontrar dificuldades em aplicar abordagens, métodos e/ou técnicas de DP, devido, principalmente, localização geográfica dos participantes.

O processo de design de interação no contexto distribuído apresenta algumas características que o torna diferente do processo de design de interação em que os participantes estão colocalizados, por exemplo, stakeholders distribuídos em várias dimensões de tempo, espaço e/ou organização [Gumm et al. 2006], necessidade de sistemas colaborativos para a realização das atividades, diferentes culturas, hábitos organizacionais e fuso horário. Como as abordagens participativas de design de interação envolvem os usuários em diversas tarefas, boas práticas participativas de design de interação colocalizado podem não ser adequadas em ambientes distribuídos.

DP em ambientes distribuídos tornou-se recentemente uma área de pesquisa denominada de Design Participativo Distribuído (DPD) [Lazarin and Almeida 2016]. DPD visa investigar técnicas e práticas envolvendo participantes geograficamente distribuídos. O DPD está preocupado com o processo de design em que os participantes têm diferentes níveis de experiência e competência e estão localizados em diferentes ambientes [Gumm et al. 2006, Lazarin and Almeida 2016, Beynon and Chan 2006].

O DP é uma mudança de atitude de "design para os usuários" para "design com usuários" [Sanders 2002], buscando o envolvimento de usuários para a colaboração no processo de design. [Baranauskas et al. 2013] propõem o envolvimento de usuários no processo de design para desempenharem o papel de criador de artefatos. Com isso, segundo os autores, é possível respeitar valores, interesses e competências dos envolvidos no produto e/ou processo de design. Isso aponta para o desafio de envolver membros da comunidade escolar no design de interação de softwares educacionais livres.

Diante disso, este artigo tem por objetivo apresentar, a partir de uma revisão de literatura, a importância e alguns desafios da adoção do DP no projeto e desenvolvimento de software educacional livre. Ao fim, são apresentados resultados preliminares de um mapeamento sistemático de literatura com o objetivo de identificar abordagens, métodos, técnicas e ferramentas para o DP de software educacional livre em ambientes distribuídos.

\section{Software Educacional Livre e Design Participativo}

Esta seção apresenta alguns conceitos fundamentais para compreender a importância do DP para a concepção e desenvolvimento de softwares educacionais livres.

\subsection{Software Educacional Livre}

[Viccari and Giraffa 1996] afirmam que "Software Educacional é um programa que visa atender necessidades e possui (ou deve possuir) objetivos pedagógicos". Assim, um software educacional refere-se a um programa de computador desenvolvido para atender aos objetivos educacionais previamente estabelecidos. Para que ele seja efetivo e esteja à altura das necessidades pedagógicas, é necessário que seu desenvolvimento conte com especialistas das áreas de educação e informática [Lucena 1994]. [Jucá 2006] afirma que um software pode ser considerado educacional quando ele é adequadamente utilizado em 
uma relação de ensino-aprendizagem. O desenvolvimento de software educacional possui como características a fundamentação em uma teoria de aprendizagem e a capacidade para a construção do conhecimento, de forma autônoma, pelo aluno [Jucá 2006].

Software livre é um termo que abrange uma diversidade de tipos de software, refere-se a programas computacionais que são distribuídos com o seu código fonte e permitem as "liberdades" de utilização, cópia, modificação e redistribuição [Reis and de Mattos Fortes 2003, Subramanyam and Xia 2008]. O termo "livre", no inglês free, é utilizado com o sentido de "liberdade" e não gratuidade.

Geralmente, o desenvolvimento de software livre é realizado por desenvolvedores distribuídos geograficamente e baseia-se em contribuições voluntárias através da Internet [Reis and de Mattos Fortes 2003]. Desenvolvedores utilizam ferramentas para coordenar e comunicar o trabalho, por exemplo, listas de discussão e sistemas de controle de versão [Reis and de Mattos Fortes 2003, Subramanyam and Xia 2008].

O sucesso de software livre resultou em milhares de projetos e produtos de software, por exemplo, Linux ${ }^{1}$, Firefox $^{2}$, GitLab $^{3}$. A educação é uma das áreas em que softwares são desenvolvidos por comunidades e projetos de software livre. Software educacional livre é distribuído com o seu código fonte e permite as liberdades de utilização, cópia, modificação e redistribuição. Esse tipo de software é desenvolvido especialmente para construir o conhecimento relativo a um conteúdo didático e favorecer os processos de ensino-aprendizagem [Jucá 2006].

A difusão da tecnologia da informação e comunicação em escolas e o aumento de softwares educacionais disponíveis na Internet são evidentes no sistema educacional. Há uma gama de softwares educacionais livres para diversas disciplinas de diferentes níveis e modalidades de ensino, por exemplo, na tabela de software educacional livre ${ }^{4}$ possui informações de cerca de 300 softwares educacionais livres. Outra iniciativa é a tabela de software educacional livre para dispositivos móveis ${ }^{5}$, que possui atualmente informações de 305 softwares.

\subsection{Design Participativo e Design Participativo Distribuído}

O DP surgiu na Escandinávia na década de 1960, por meio de uma parceria entre acadêmicos e sindicatos. Desde então, vários projetos tiveram a iniciativa de envolver os usuários no design. O DP tornou-se uma abordagem importante para pesquisadores interessados em IHC e outras áreas [Rogers et al. 2013, Spinizzi 2005].

Segundo [Muller et al. 1997], não há uma definição única de DP que satisfaça todos os pesquisadores e praticantes nessa área. O DP busca envolver usuários no processo de design e, assim, fazer com que a voz do usuário reflita em contribuições para o projeto e desenvolvimento de produtos que eles utilizarão [Rogers et al. 2013, Muller et al. 1997]. [Kensing and Blomberg 1998] afirmam que DP é uma área de pesquisa e uma prática em evolução entre profissionais de design, em que pesquisadores buscam condições para a

\footnotetext{
${ }^{1}$ https://www.linuxfoundation.org//

${ }^{2}$ https://www.mozilla.org/pt-BR/firefox/new//

${ }^{3}$ https://about.gitlab.com//

${ }^{4}$ https://goo.gl/97Eu8h

${ }^{5}$ https: / / goo.gl/35iB3y
} 
VI Congresso Brasileiro de Informática na Educação (CBIE 2017)

Anais dos Workshops do VI Congresso Brasileiro de Informática na Educação (WCBIE 2017)

participação dos usuários no design e também na introdução de sistemas baseados em computador no ambiente de trabalho.

Pesquisadores da área de IHC têm apontado a importância de considerar o usuário como elemento central no processo de concepção de sistemas interativos. [Melo and Baranauskas 2006] afirmam que diferentes paradigmas de design surgiram nos últimos anos, variando as estratégias para considerar o usuário no processo de design, por exemplo, o Design Centrado no Usuário, o Design Participativo, entre outros. As autoras afirmam que não existem dúvidas de que o usuário deve ser considerado no processo de design.

[Baranauskas et al. 2013] afirmam que o conceito de usuário, em que alguns designers se colocam na posição de representantes dos usuários, não deixa espaço para a pessoa definir-se enquanto parte interessada em um produto de design e agir em favor de seus próprios interesses. Os autores propõem o modelo semioparticipativo, no qual não cabe esse conceito de usuário e é substituído por "partes interessadas" no produto e processo de design, como forma de respeitar valores, interesses e competências daqueles envolvidos no produto e/ou processo de design.

O DP se torna desafiador quando os stakeholders estão geograficamente distribuídos [Danielsson et al. 2006]. Assim, a área de pesquisa de DPD surgiu da necessidade de tornar possível o DP em ambientes distribuídos de desenvolvimento de software. [Beynon and Chan 2006, p. 1, tradução nossa] afirmam que "O design participativo distribuído (DPD) está preocupado com os processos de design em que os stakeholders têm diferentes níveis de experiência e competência e estão localizados em diferentes ambientes".

Diante desse contexto, verifica-se a importância e o desafio de envolver membros da comunidade escolar para colaborarem no projeto e desenvolvimento de softwares educacionais livres. A colaboração desses usuários na concepção e desenvolvimento de software educacional livre pode contribuir no atendimento às expectativas e objetivos dos usuários, respeitando seus interesses e valores. Além disso, essa colaboração pode também contribuir no atendimento aos objetivos e necessidades educacionais.

\section{A Importância do Design Participativo no Desenvolvimento de Software Educacional Livre}

Apesar do aumento no número de projetos, publicações e o sucesso de software livre, a literatura científica indica alguns problemas de IHC relacionados aos produtos e processos de desenvolvimento de software livre, a citar: existem problemas relacionados à usabilidade e à acessibilidade em software livre [Hedberg and Iivari 2009, Alves et al. 2014]; apesar do impacto generalizado de software livre na área de computação, a comunidade de IHC tem realizado poucos estudos sobre software livre e existem poucas parcerias com a comunidade de software livre [Bach and Terry 2010]; aspectos de usabilidade são considerados, em grande parte, tardiamente em projetos de software livre [Schwartz and Gunn 2009]; a maioria dos projetos de software livre tem requisitos fundamentalmente definidos pelos seus autores e pouca ênfase é dada à usabilidade [Reis and de Mattos Fortes 2003]. Acredita-se que muitos desses problemas também podem estar relacionados ao produto e/ou processo de desenvolvimento de software educacional livre. Somado a isso, destaca-se que os softwares educacionais livres devem 
VI Congresso Brasileiro de Informática na Educação (CBIE 2017)

Anais dos Workshops do VI Congresso Brasileiro de Informática na Educação (WCBIE 2017)

atender aos objetivos educacionais e às necessidades pedagógicas. Assim, a colaboração da comunidade escolar no desenvolvimento de software educacional livre é importante para diminuir os problemas relacionados à $\mathrm{IHC}$ e essencial para o atendimento às necessidades pedagógicas e aos objetivos educacionais, estimulando um ambiente de maior cooperação.

[Haskel and Graham 2016] afirmam que software livre é uma prática participativa que contrasta com DP de muitas maneiras. As comunidades de software livre podem ser fortes, mas estão estreitamente focadas aos interesses e atividades dos desenvolvedores. Além disso, a interação entre desenvolvedores e usuários pode ser fraca e desestruturada. Apesar disso, DP e software livre compartilham alguns valores importantes em seu compromisso comunitário e o benefício do esforço colaborativo. As liberdades inerentes ao software livre permitem a aprendizagem, prototipação, colaboração e experimentação, todas atividades também inerentes ao DP [Haskel and Graham 2016].

Dentre as características do desenvolvimento de software livre está o ambiente colaborativo e comunitário, no qual os participantes podem contribuir de forma descentralizada por meio da Internet. Isso torna possível um maior envolvimento e diversidade de usuários finais no desenvolvimento de software educacional livre. Assim, acreditase que membros da comunidade escolar geograficamente distribuídos, incluindo pessoas com deficiência e idosos, poderiam contribuir no desenvolvimento de software educacional livre.

Algumas iniciativas envolveram as áreas de DP e software educacional, por exemplo, um framework semioparticipativo para o design de interação de softwares educacionais chamado SPIDe [Rosa and Matos 2016]. No entanto, o estudo focou em projetos onde os stakeholders estavam colocalizados. Considerando os projetos de software educacional livre, no caso em que os stakeholders podem estar distribuídos geograficamente, verifica-se a necessidade de abordagens de DP que envolvam a colaboração de forma distribuída.

\section{Desafios de Design Participativo Distribuído em Software Educacional Livre}

Um dos desafios de DPD está relacionado à falta de abordagens, métodos e técnicas de DP para projetos de software em ambientes distribuídos [Gumm et al. 2006, Lazarin and Almeida 2016]. As abordagens de DP raramente levam em consideração a distribuição geográfica dos stakeholders e, geralmente, essas abordagens são baseadas na possibilidade de reuniões presenciais. Apesar disso, é crescente o número de projetos de software desenvolvidos em ambientes distribuídos, por exemplo, software livre e open source [Gumm et al. 2006]. [Lazarin and Almeida 2016] afirmam que a maioria das técnicas de DP é explorada por meio de práticas presenciais e síncronas. No entanto, essas técnicas não são adequadas em diversos contextos, especialmente para situações em que os stakeholders estão distribuídos geograficamente. Diante disso, o DP em projetos de softwares educacionais livres, em ambientes distribuídos, é um desafio, pois a falta de abordagens, métodos e técnicas de DP para ambientes em que os stakeholders estão distribuídos geograficamente pode dificultar o envolvimento de membros da comunidade escolar. Isso mostra que novas pesquisas e propostas para o DP em ambientes distribuídos de desenvolvimento de software educacional livre são necessárias. 
A comunicação também é um desafio para DP em ambientes distribuídos. O acesso à informação é um requisito crítico em DP. A distribuição física dos stakeholders dificulta a comunicação informal, o que pode conduzir para a falta de informação no projeto. Além disso, a separação de tarefas dificulta o trabalho sincronizado, reuniões presenciais, a cooperação e aprendizado mútuo entre os stakeholders. A construção da confiança entre os stakeholders distribuídos geograficamente também é um desafio. A dificuldade relacionada à confiança pode prejudicar o processo de DP [Gumm 2006].

Segundo [Gumm et al. 2006], um dos desafios no processo de design no contexto distribuído é o envolvimento de usuários. As autoras defendem a aplicação de uma mistura de métodos para a participação de usuários no processo de design distribuído e evidenciam a necessidade de mais métodos para abordar a distribuição e as necessidades de diferentes usuários. Isso mostra que o envolvimento de membros da comunidade escolar em projetos de software educacional livre também pode ser um desafio. A adequação para o contexto distribuído de técnicas e práticas de DP colocalizado e a utilização de sistemas colaborativos podem contribuir nesse desafio.

Ainda sobre o desafio de envolvimento de usuários no DP em ambientes distribuídos de desenvolvimento de software, de acordo com uma pesquisa realizada sobre desenvolvedores de software livre e open source, 98\% dos desenvolvedores entrevistados eram do sexo masculino e a idade média era 27 anos [Ghosh et al. 2002]. [Davidson 2013] cita que a falta de diversidade em software livre e open source pode significar que as vozes dos usuários finais não estejam sendo ouvidas. Uma pesquisa sobre acessibilidade no desenvolvimento de software livre mostrou que em apenas $27 \%$ dos projetos de software livre as pessoas com deficiência e/ou idosos participaram do desenvolvimento de software livre [Alves et al. 2014]. O desenvolvimento de mecanismos computacionais que considerem a grande diversidade de pessoas têm potencial para facilitar a interação e a colaboração, mas caso desenvolvido sem considerar as diferenças entre as pessoas, podem resultar em situações segregadoras [Baranauskas et al. 2013]. Assim, há evidências de que o envolvimento e a diversidade de usuários finais no projeto e desenvolvimento de software educacional livre também são desafios de DP no contexto distribuído.

\section{Mapeamento Sistemático de Literatura: resultados preliminares}

A comunidade científica busca elementos que contribuam para a superação dos desafios de pesquisa em Ciência da Computação e Educação, especialmente no tocante ao acesso participativo e universal do cidadão brasileiro ao conhecimento [Baranauskas and Souza 2006]. Verifica-se a necessidade de explorar sistematicamente quais são as (os) abordagens, métodos, técnicas e ferramentas para o DP de software educacional livre no contexto distribuído. Com isso, será possível identificar os gaps e a direção em que pesquisas na área de DPD devem seguir. Nesta seção é apresentada uma proposta preliminar de um mapeamento sistemático de literatura para identificar abordagens, métodos, técnicas e ferramentas para o DP em ambientes distribuídos de desenvolvimento de software educacional livre.

O mapeamento sistemático será baseado no modelo de protocolo apresentado por [Wohlin et al. 2012] e nas recomendações fornecidas por [Kitchenham and Charters 2007]. O protocolo deste mapeamento está disponível 
VI Congresso Brasileiro de Informática na Educação (CBIE 2017)

Anais dos Workshops do VI Congresso Brasileiro de Informática na Educação (WCBIE 2017)

em https://goo.gl/ECjEe8 .

\subsection{Questão e Subquestões de Pesquisa}

O mapeamento sistemático busca responder a seguinte questão de pesquisa:

Quais são as (os) abordagens, métodos, técnicas e ferramentas para o design participativo em ambiente distribuído de desenvolvimento de software educacional livre?

A questão de pesquisa principal foi decomposta nas seguintes subquestões de pesquisa mais detalhadas:

sub-QP1 - a (o) abordagem, método, técnica ou ferramenta para o DP foi concebida/aplicada (o) paralem ambiente distribuído ou foi concebida/aplicada (o) um (a) paralem ambiente colocalizado?

sub-QP2 - a (o) abordagem, método, técnica ou ferramenta para o DP está relacionada (o) a qual (is) atividade (s) do processo de design de interação?

sub-QP3 - a (o) abordagem, método, técnica ou ferramenta para o DP proposta (o) foi validada (o) por meio de estudos empíricos?

sub-QP4 - quais os benefícios e as limitações ao adotar a (o) abordagem, método, técnica ou ferramenta de DP em ambientes distribuídos de desenvolvimento de software?

\subsection{Processo de Seleção de Estudos}

As seguintes etapas estão sendo realizadas na seleção de estudos:

1. busca de estudos nas bibliotecas digitais;

2. seleção de estudos preliminar ( $1^{\circ}$ filtro: leitura de título e abstract);

3. seleção de estudos ( $2^{\circ}$ filtro: leitura completa dos estudos selecionados na etapa 2);

4. extração de dados dos estudos incluídos;

5. tabulação e análise dos estudos selecionados restantes.

\subsection{Estratégia de Busca}

As bibliotecas digitais utilizadas para pesquisa de estudos primários são: ACM Digital Library $^{6}$, Engineering Village ${ }^{7}$, IEEE Xplore Digital Library ${ }^{8}$, e ScienceDirect ${ }^{9}$. As bibliotecas digitais foram escolhidas por indexarem a maioria dos veículos qualificados na área de Ciência da Computação e devido ao acesso livre e gratuito aos periódicos no âmbito da Universidade Federal da Bahia (UFBA).

As seguintes palavras-chaves serão utilizadas: "distributed participatory design of educational software", "online participatory design of educational software", "participatory design in free educational software", "participatory design in open source educational software".

De acordo com as palavras-chaves, foi definida a string a ser utilizada nas buscas das bibliotecas digitais. A string foi definida apenas em inglês, conforme a seguir:

\footnotetext{
${ }^{6}$ http: //dl.acm.org/

${ }^{7}$ http: / / www.engineeringvillage.com/

${ }^{8}$ http: //ieeexplore.ieee.org/Xplore/home.jsp

${ }^{9}$ http: //www.sciencedirect.com/
} 
VI Congresso Brasileiro de Informática na Educação (CBIE 2017)

Anais dos Workshops do VI Congresso Brasileiro de Informática na Educação (WCBIE 2017)

("participatory design" AND ("free software" OR "open source" OR distributed OR online) AND (education OR educational))

\subsection{Critérios de Inclusão e Exclusão}

Para a inclusão de um estudo será determinada sua relevância em relação à questão de pesquisa.

Os estudos que preencherem os seguintes critérios de inclusão serão incluídos:

- estudos que apresentam abordagem, método, técnica e/ou ferramenta para o DP em ambientes distribuídos de desenvolvimento de software educacional livre;

Serão excluídos os estudos que preencherem pelo menos um dos seguintes critérios de exclusão:

- artigos introdutórios para questões especiais e resumo de eventos, por exemplo, workshops;

- artigos que não estejam escritos em inglês;

- artigos duplicados;

- artigos sem acesso disponível para visualização e/ou download do texto completo, especialmente em casos em que os estudos são pagos ou não disponibilizados na biblioteca digital;

- trabalhos que não são artigos de conferência ou de periódico.

\subsection{Resultados Preliminares do Mapeamento Sistemático}

Com a aplicação da string de busca citada na subseção 5.3 foram encontrados 108 estudos, separados por fonte, da seguinte forma: IEEE Xplore, 19 estudos; ACM Digital Library, 29 estudos; ScienceDirect, 3 estudos; Engineering Village, 57 estudos.

A próxima etapa do mapeamento sistemático será efetuar a leitura dos títulos e $a b$ stracts dos trabalhos encontrados. Assim, os trabalhos considerados relevantes, de acordo com os critérios de inclusão e exclusão estabelecidos, serão selecionados. Posteriormente, será realizada a seleção de estudos por meio da leitura completa dos estudos selecionados na etapa de seleção preliminar, extração de dados dos estudos incluídos e tabulação e análise dos estudos primários incluídos.

\section{Considerações Finais}

Este artigo evidenciou a importância da colaboração de membros da comunidade escolar no projeto e desenvolvimento de software educacional livre. Para isso, apresentou a abordagem de DP, seus conceitos, desafios e a importância do DP para software educacional livre. Além disso, foi apresentado um mapeamento sistemático de literatura em andamento que visa identificar abordagens, métodos, técnicas e ferramentas para o DP em ambiente distribuído de desenvolvimento de software educacional livre.

A partir dos desafios verificados, gaps identificados e problemas de IHC em software livre, pretende-se avançar na pesquisa sobre esse assunto. Enfatiza-se a relevância do tema, destaca-se a importância da colaboração de usuários e a aplicação de abordagens, métodos e técnicas participativas no desenvolvimento de software educacional livre. 
VI Congresso Brasileiro de Informática na Educação (CBIE 2017)

Anais dos Workshops do VI Congresso Brasileiro de Informática na Educação (WCBIE 2017)

Diante disso, o próximo passo da pesquisa será continuar o mapeamento sistemático proposto neste artigo. Após isso, deve-se conceber e avaliar um modelo de processo de design de interação que apoiará a colaboração de stakeholders geograficamente distribuídos no desenvolvimento de software educacional livre. Por fim, espera-se que esse estudo seja um esforço inicial para que novas pesquisas e propostas relacionadas a esse tema sejam realizadas.

\section{References}

Alves, D. D., Cagnin, M. I., and Paiva, D. M. B. (2014). Accessibility in development of free software projects. In Computing Conference (CLEI), 2014 XL Latin American, pages 1-12. IEEE.

Bach, P. M. and Terry, M. (2010). The future of floss in chi research and practice. In CHI'10 Extended Abstracts on Human Factors in Computing Systems, pages 44734476. ACM.

Baranauskas, M. C. C., Martins, M. C., and Valente, J. A. (2013). Codesign de redes digitais: Tecnologia e educação a serviço da inclusão social. Penso Editora.

Baranauskas, M. C. C. and Souza, C. S. d. (2006). Desafio 4: Acesso participativo e universal do cidadão brasileiro ao conhecimento. Computação Brasil, 7(23).

Beynon, M. and Chan, Z. E. (2006). A conception of computing technology better suited to distributed participatory design. In NordiCHI Workshop on Distributed Participatory Design.

Danielsson, K., Naghsh, A. M., and Dearden, A. (2006). Distributed participatory design. In Workshop at the 4th Nordic Conference on Human-Computer Interaction.

Davidson, J. L. (2013). Involving older adults in the design and development of free/open source software. In Visual Languages and Human-Centric Computing (VL/HCC), 2013 IEEE Symposium on, pages 177-178. IEEE.

Ghosh, R. A., Glott, R., Krieger, B., and Robles, G. (2002). Free/libre and open source software: Survey and study.

Gumm, D. C. (2006). Distributed participatory design: An inherent paradoxon? Proc. of IRIS29.

Gumm, D. C., Janneck, M., and Finck, M. (2006). Distributed participatory design-a case study. In Proceedings of the DPD Workshop at NordiCHI, volume 2.

Haskel, L. and Graham, P. (2016). Whats gnu got to do with it?: participatory design, infrastructuring and free/open source software. In Proceedings of the 14th Participatory Design Conference: Short Papers, Interactive Exhibitions, Workshops-Volume 2, pages 17-20. ACM.

Hedberg, H. and Iivari, N. (2009). Integrating hci specialists into open source software development projects. Open Source Ecosystems: Diverse Communities Interacting, pages 251-263.

Jucá, S. C. S. (2006). A relevância dos softwares educativos na educação profissional. Ciências \& Cognição, 8:22-28. 
VI Congresso Brasileiro de Informática na Educação (CBIE 2017)

Anais dos Workshops do VI Congresso Brasileiro de Informática na Educação (WCBIE 2017)

Kensing, F. and Blomberg, J. (1998). Participatory design: Issues and concerns. Computer Supported Cooperative Work (CSCW), 7(3-4):167-185.

Kitchenham, B. and Charters, S. (2007). Guidelines for performing systematic literature reviews in software engineering. In Technical report, Ver. 2.3 EBSE Technical Report.

Lazarin, C. A. J. and Almeida, L. D. A. (2016). Distributed participatory design webbased groupware: gathering requirements through braindraw. In Proceedings of the 15th Brazilian Symposium on Human Factors in Computer Systems, page 3. ACM.

Lucena, M. W. F. P. (1994). O uso das tecnologias da informática para o desenvolvimento da educação. Rio de Janeiro: COPPE/UFRJ.

Melo, A. M. and Baranauskas, M. C. C. (2006). Design para a inclusão: desafios e proposta. In Proceedings of VII Brazilian symposium on Human factors in computing systems, pages 11-20. ACM.

Muller, M. J., Haslwanter, J. H., and Dayton, T. (1997). Participatory practices in the software lifecycle. Handbook of human-computer interaction, 2:255-297.

Reis, C. R. and de Mattos Fortes, R. P. (2003). Caracterização de um Processo de Software para Projetos de Software Livre. $\mathrm{PhD}$ thesis, $\mathrm{PhD}$ thesis, University of Sao Paulo, Brazil.

Rogers, Y., Sharp, H., and Preece, J. (2013). Design de interação: além da interação humano-computador. Bookman.

Rosa, J. and Matos, E. (2016). Semio-participatory framework for interaction design of educational software. In Proceedings of the 15th Brazilian Symposium on Human Factors in Computer Systems, page 33. ACM.

Sanders, E. B.-N. (2002). From user-centered to participatory design approaches. Design and the social sciences: Making connections, 1(8).

Schwartz, D. and Gunn, A. (2009). Integrating user experience into free/libre open source software: Chi 2009 special interest group. In CHI'09 Extended Abstracts on Human Factors in Computing Systems, pages 2739-2742. ACM.

Spinizzi, C. (2005). The methodology of participatory design. technical communication.

Stallman, R. (1998). Why free software is better than open source. Disponível em: http://www.fsf.org/philosophy/free-software-for-freedom.html .Acessado: 06 fev. 2017.

Subramanyam, R. and Xia, M. (2008). Free/libre open source software development in developing and developed countries: A conceptual framework with an exploratory study. Decision support systems, 46(1):173-186.

Viccari, R. M. and Giraffa, L. M. M. (1996). Sistemas tutores inteligentes: abordagem tradicional x abordagem de agentes. In Berlim: Springer Verlag, page 89. XIII SBIA Simpósio Brasileiro de Inteligência Artificial.

Wohlin, C., Runeson, P., Höst, M., Ohlsson, M. C., Regnell, B., and Wesslén, A. (2012). Experimentation in software engineering. Springer Science \& Business Media. 